\title{
Single-File Escape of Colloidal Particles from Microfluidic Channels
}

\author{
Emanuele Locatelli ${ }^{*}$ and Matteo Pierno ${ }^{\dagger}$ \\ Dipartimento di Fisica e Astronomia "G. Galilei” (DFA) and Sezione CNISM, Università di Padova, \\ Via Marzolo 8, 35131 Padova, Italy \\ Fulvio Baldovin ${ }^{\ddagger}$ and Enzo Orlandini ${ }^{\S}$ \\ Dipartimento di Fisica e Astronomia “G. Galilei” (DFA), Sezione INFN and Sezione CNISM, Università di Padova, \\ Via Marzolo 8, 35131 Padova, Italy \\ Yizhou Tan and Stefano Pagliara \\ Cavendish Laboratory, Cambridge CB3OHE, United Kingdom \\ (Received 12 February 2016; revised manuscript received 6 May 2016; published 12 July 2016)
}

\begin{abstract}
Single-file diffusion is a ubiquitous physical process exploited by living and synthetic systems to exchange molecules with their environment. It is paramount to quantify the escape time needed for single files of particles to exit from constraining synthetic channels and biological pores. This quantity depends on complex cooperative effects, whose predominance can only be established through a strict comparison between theory and experiments. By using colloidal particles, optical manipulation, microfluidics, digital microscopy, and theoretical analysis we uncover the self-similar character of the escape process and provide closed-formula evaluations of the escape time. We find that the escape time scales inversely with the diffusion coefficient of the last particle to leave the channel. Importantly, we find that at the investigated microscale, bias forces as tiny as $10^{-15} \mathrm{~N}$ determine the magnitude of the escape time by drastically reducing interparticle collisions. Our findings provide crucial guidelines to optimize the design of micro- and nanodevices for a variety of applications including drug delivery, particle filtering, and transport in geometrical constrictions.
\end{abstract}

DOI: 10.1103/PhysRevLett.117.038001

Living and synthetic systems exploit a variety of pores or channels at the micro- and nanoscale, to transport particles and molecules [1,2]. When the pore or channel cross section is close to that of the particles, these are no longer able to pass each other, a phenomenon known as single-file diffusion (SFD). SFD plays a role in numerous processes such as the diffusion of ions or water molecules in transmembrane proteins [3-8], the diffusion of adsorbate molecules in zeolites [9-11], water diffusion in nanotubes [12], colloidal particle diffusion in one-dimensional (1D) channels [13-15], drug delivery through nanofluidic devices [16], protein sliding along DNA [17], and charge carrier migration in polymer and superionic conductors [18,19]. SFD is a fascinating process, since it does not obey Fick's laws. As the sequence of particles remains unaffected over time, anomalous behavior in fact characterizes SFD systems. Specifically, it has been shown that single-file interactions imply that the mean square displacement (MSD) of a tagged particle scales as $t^{1 / 2}$ [20].

Recently, a wealth of theoretical approaches to analyze SFD has been developed [18,21-35] and the dependence of the MSD on time has been experimentally investigated for many SFD systems: (i) At the nanoscale with measurements on zeolites [9,10] and single-walled carbon nanotubes [12] via nuclear magnetic resonance; (ii) at the microscale, on colloidal suspensions confined in circular trenches [14], in $1 \mathrm{D}$ circular channels created by means of scanning optical tweezers [13] in narrow straight grooves [15,36] and in narrow microfluidic channels [37]; and (iii) at the millimeter scale, on macroscopic charged metallic balls electrostatically interacting and confined in a circular channel [38]. So far, both experimental and theoretical approaches have been focused on the temporal dependence of the MSD of the particles inside the channels. On the contrary, little is known about the escape process of single-file particles out of a narrow channel, a key issue in the analysis of diffusive transport in compartmentalized systems [39].

In this Letter we address this problem with a combined experimental and theoretical investigation that allows us to dissect the single contributions of the different cooperative effects involved in the escape process. The experiments are based on colloidal particles in microfluidics [40-43], holographic optical tweezers (HOTs) [44-47], and digital video microscopy [48]. This setup allows us to monitor the position of colloidal particles in single file within arrays of microfluidic channels with different lengths [49-52]. We measure the survival probability for the last particle to leave each channel and the mean escape time needed for all the particles to leave the channel. We compare these measurements with analytical predictions developed on the basis of the reflection principle method [21,35]. This allows us to successfully validate closed formulas for the estimation of the mean escape time, which can be used for quantitative assessment in widespread single-file conditions, on both 
living and synthetic model systems [2,5]. We find that (i) the escape process of $N$ particles can be entirely described in terms of the survival probability of the last particle to leave the channel, (ii) the escape time scales inversely with the diffusion coefficient of a single particle in the channel, and (iii) bias forces as tiny as $10^{-15} \mathrm{~N}$ determine the magnitude of the escape time by drastically reducing interparticle collisions and switching off excluded-volume effects.

Microfluidic devices consisting of two 3D baths with a depth of $16 \mu \mathrm{m}$ separated by a polydimethylsiloxane barrier and connected by an array of microfluidic channels were fabricated as previously reported [53,54]. The channels have cross-section dimensions close to $1 \mu \mathrm{m}$, and length $L_{c}$ of 4.7 [Fig. 1(a)], 5, 6, 7, 9.6, or $12 \mu \mathrm{m}$. For full details about the channel geometries, see Table S1 in Supplemental Material [55]. The baths are filled with polystyrene spherical particles with radius $R=(252.5 \pm 4)$ or $(310 \pm 5) \mathrm{nm}$ dispersed in a $5 \mathrm{mM} \mathrm{KCl}$ salt solution.
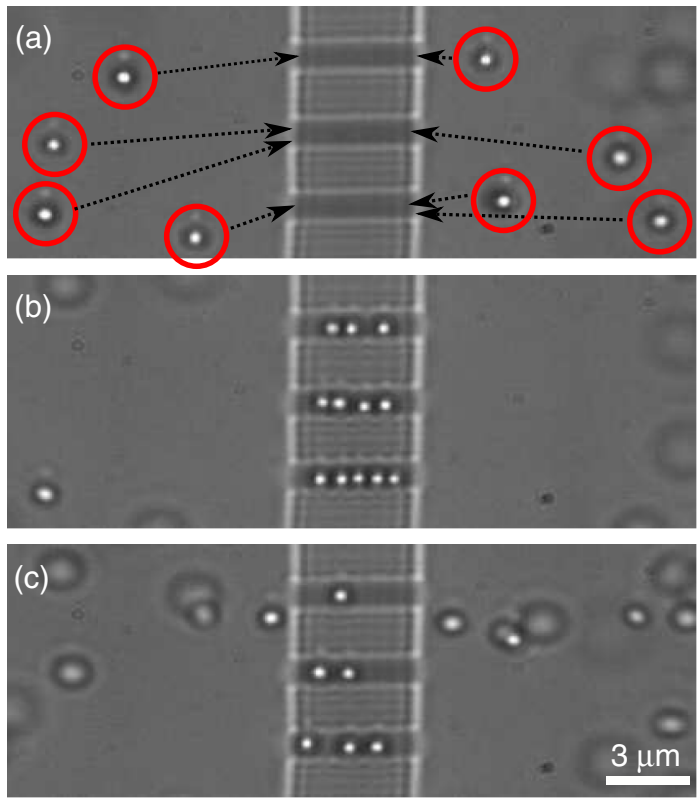

FIG. 1. Filling and escape processes of colloidal particles from an array of microfluidic channels. (a) Two three-dimensional (3D) baths are filled with freely diffusing particles. The baths are connected by an array of microfluidic channels with a similar cross section and length $L_{c}$ of $4.7 \mu \mathrm{m}$. Eight optical traps are generated via holographic optical tweezers and positioned in the 3D baths. Trapped particles (highlighted with circles) are independently dragged and accurately positioned in the microfluidic channels (dotted arrows depict the dragging trajectories until the channel entrances). (b) Afterward, four more optical traps are generated in the baths and used to drag four more particles in the channels so that an array of $N=3,4$, and 5 single-file particles is generated in the top, central, and bottom channel, respectively. Particles are then released by switching off the trapping laser at $t=0$. (c) Exemplary snapshot of the escape processes at $t=18 \mathrm{~s}$. The escape processes are followed until all the particles in the array of channels have escaped to the $3 \mathrm{D}$ baths.
The Debye length associated with the particles is around $6 \mathrm{~nm}$, much smaller than the particle radii, thus justifying the assumption of hard-sphere interactions in the theoretical model below. We use a custom-made HOTs setup [49] to generate multiple optical traps in the 3D baths, where particles freely diffuse [Fig. 1(a) and video S1]. Upon trapping, particles are independently dragged and positioned in the microfluidic channels with an accuracy down to $100 \mathrm{~nm}$ [Fig. 1(b)], generating an array of single-file particles with different initial numbers $N=3,4$, and 5 in each channel [Fig. 1(b)]. Once the array is completed, all particles are released at the same time by switching off the trapping laser. The escape processes from the different channels are followed at all times. Figure 1(c) reports an exemplary snapshot of the escape processes 18 seconds after the release instant. Each escape process is repeated at least 50 times. Overall, we investigate escape processes from 13 different channels involving the trapping and escape of more than four thousand particles (Table S1). We track the position of each particle in each channel at all times by using a custom-written interactive data language routine based on a standard particle tracking approach [48]. In each experiment we measure $J_{r-l}$ [see Table $S 1$ and Figs. S1(a) and S1(b)], the average difference in the number of particles exiting from each channel to the right and left baths $[54,59]$. This allows us to identify the intensity of any external bias force $F_{e}$ down to the scale of $10^{-15} \mathrm{~N}$ and its contribution to the escape process via the adimensional ratio $\left(F_{e} L_{c}\right) /\left(k_{B} T\right)$ (see Supplemental Material [55]).

We measure the escape time $\tau$, i.e., the time required for all the particles to exit from the channel to the baths. Detailed information about the escape process can be obtained in terms of the survival probability, experimentally defined as

$$
S_{1}(t) \equiv \frac{M_{t}}{M}=\operatorname{Prob}\{\tau>t\}
$$

where $M$ is the number of repeats of the same escape process and $M_{t}$ is the subset of repeats for which $\tau$ is larger than $t$. The corresponding errors are evaluated as $\sqrt{M_{t}} / M$. From $S_{1}(t)$ one can estimate the mean escape time $T_{1}=\int_{0}^{+\infty} d t S_{1}(t)$. Note that both $S_{1}(t)$ and $T_{1}$ depend on several experimental parameters, namely, the length of the channel $L_{c}$, the number of initial particles $N$, and the width $L_{0} \leq L_{c}$ of the distribution of the initial positions of the particles when these are released by switching off the trapping laser (Fig. S2). Moreover, a systematic external bias force $F_{e}$ can also affect $S_{1}(t)$.

In order to gain information on the different physical mechanisms contributing to the escape process, we carry out an analytical evaluation of $S_{1}(t)$ and $T_{1}$ based on the reflection principle method [21,35] (full details are reported in Supplemental Material [55]). The starting quantity is the conditional probability $S_{n}\left(t \mid m, L_{c}, L_{0}, \Gamma\right)$. This is the 
survival probability of at least $n$ particles, given that at time $t=0 m \geq n$ were uniformly distributed within the interval $\left[-L_{0} / 2, L_{0} / 2\right]$ of a channel of total length $L_{c} \geq L_{0}$. The parameter $\Gamma \equiv F_{e} / 2 k_{B} T$ quantifies the bias. With $n=1$ and $m=N, S_{n}\left(t \mid m, L_{c}, L_{0}, \Gamma\right)$ corresponds to the experimental observable $S_{1}(t)$. In the limit of pointlike particles the following relation holds true [35]:

$$
1-S_{1}\left(t \mid N, L_{c}, L_{0}, \Gamma\right)=\left[1-S_{1}\left(t \mid 1, L_{c}, L_{0}, \Gamma\right)\right]^{N} .
$$

The analytical expression for the single-particle survival probability in the presence of a bias, $S_{1}\left(t \mid 1, L_{c}, L_{0}, \Gamma\right)$, is reported in Eq. (6) of the Supplemental Material [55]. By inserting this formula in Eq. (2), we obtain an analytical expression for $S_{1}(t)$. Importantly, Eq. (2) implies that the multiparticle escape process can be mapped onto the escape process of many independent particles. Indeed, the multiparticle escape from the channel can be considered as a collective process where the order in which particles escape from the channel can be neglected. Therefore, in our model we do not tag any of the particles, thus neglecting collisions (equal-mass particles exchange their velocities in 1D elastic collisions) while assuming independent pointlike particles. Intuitively, while collisions hamper the diffusion of the particles in the center of the single file, they simultaneously contribute to expel those at the edges of the channel, the two effects thereby canceling each other. As a consequence the $S_{1}(t)$ values calculated according to Eq. (2) [lines in Fig. 2(a)] favorably compare with the experimentally measured values [symbols in Fig. 2(a)]. Remarkably, by rescaling $t$ to $t / T_{1}\left(N, L_{c}\right)$ both the experimental data and the analytical expressions collapse onto the same curve [Fig. 2(b) and Fig. S4]. This suggests that $T_{1}$ can be used as a scaling parameter for characterizing the self-similarity of the escape process.

An analytical expression for $T_{1}$ is obtained by applying the binomial formula to the integral of Eq. (2),

$$
\begin{aligned}
T_{1}\left(N, L_{c}, L_{0}, \Gamma\right)= & \sum_{k=1}^{N}\left\{(-1)^{k+1}\left(\begin{array}{l}
N \\
k
\end{array}\right)\right. \\
& \left.\cdot \int_{0}^{+\infty} d t\left[S_{1}\left(t \mid 1, L_{c}, L_{0}, \Gamma\right)\right]^{k}\right\} . \\
= & \frac{L_{c}^{2}}{D_{1}} g\left(N, L_{c}, L_{0}, \Gamma\right),
\end{aligned}
$$

where

$$
\begin{aligned}
g\left(N, L_{c}, L_{0}, \Gamma\right) \equiv & \sum_{k=1}^{N}\left\{(-1)^{k+1}\left(\begin{array}{c}
N \\
k
\end{array}\right)\right. \\
& \left.\cdot \int_{0}^{+\infty} d t^{\prime}\left[S_{1}\left(t^{\prime} \mid 1, L_{c}, L_{0}, \Gamma\right)\right]^{k}\right\},
\end{aligned}
$$

and the change of variable to adimensional time $t^{\prime} \equiv$ $t D_{1} / L_{c}^{2}$ has been performed. The interesting feature of

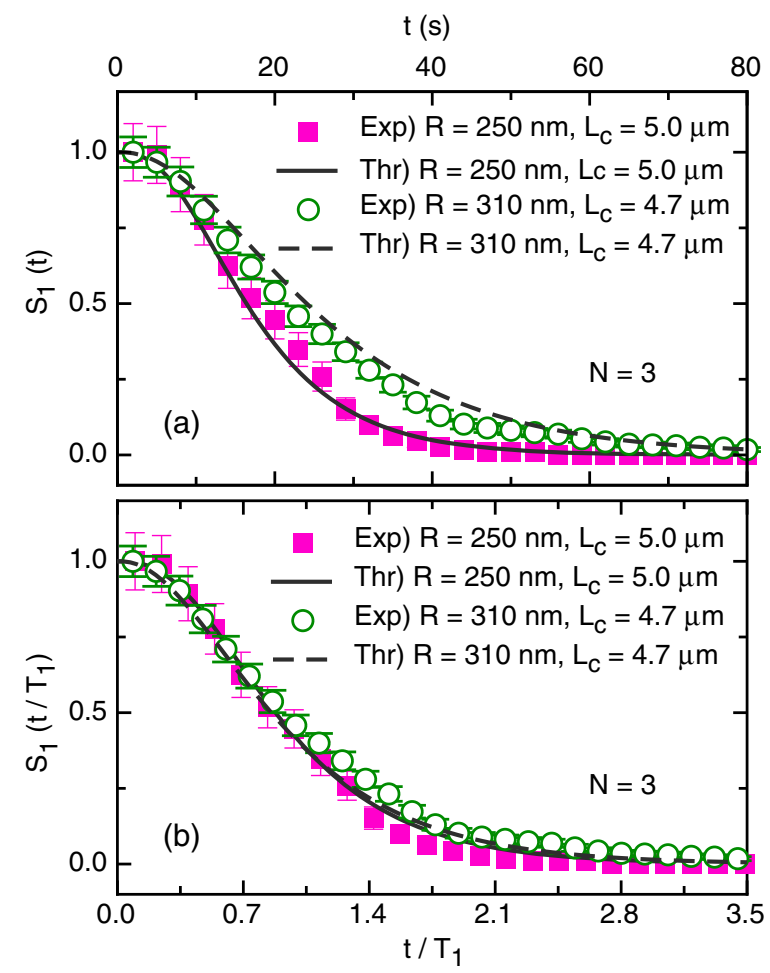

FIG. 2. (a) Time dependence of the escape-process survival probability $S_{1}(t)$ of $N=3$ particles in single file. Squares and circles show the experimental results. Solid and dashed curves refer to theoretical predictions calculated according to Eq. (2). (b) Dependence of $S_{1}$ on $t / T_{1}$ for the same quantities plotted in panel (a). Further plots are reported in Supplemental Material [55].

Eq. (3) is that it expresses a collective behavior of $N$ particles in single file (lhs) only in terms of the survival probability of a single particle in the channel (rhs). Importantly, $T_{1}$ scales inversely with the diffusion coefficient $D_{1}$ of the last particle to leave the microfluidic channel.

To validate this theoretical description, we experimentally measure the mean escape time $T_{1}$ and the diffusion coefficient $D_{1}$ of the last colloidal particle in the channel, after all the other particles have left the channel. In this way, the measurement of $D_{1}$ [reported in Table S1 and Fig. S1(c)] is not affected by particle-particle interactions. We evaluate $D_{1}$ through the MSD as $D_{1}=\operatorname{MSD}(n \Delta t) /(2 n \Delta t)$, where $\Delta t$ is the lag time between consecutive frames and $n=1,2, \ldots, N_{t}-1$ are the last particle trajectory points. In agreement with previous findings [51,54], due to particlechannel interactions and hydrodynamic effects, $D_{1}$ is found to be lower on average by about $1 / 5$ than the diffusion coefficient measured in the bulk (Table S1). With experimental conditions very similar to the ones used in the present study, it has also been shown [51] that $D_{1}$ is approximately uniform throughout the entire channel length, the transition region between bulk and channel being located beyond the channel ends. Furthermore, in 
the Supplemental Material [55] we outline a simple theoretical discussion on the impact that particle-channel interactions have on $D_{1}$ at the channel entrances.

Under small external biases $F_{e}<10^{-15} \mathrm{~N}$, the theoretical estimations of $T_{1}$ calculated according to Eq. (3) [mathematical symbols in Fig. 3(a)] slightly overestimate the experimental values (full symbols). In such cases, a better description of the experimental findings is given by an effective theory that accounts for excluded volume effects between colloidal particles. In the limit of weak bias $\Gamma L_{c} \ll 1$ ( or $k_{B} T \gg F_{e} L_{c}$ ), the single-particle survival probability only depends on the ratio $L_{0} / L_{c}$ and Eq. (3) simplifies into

$$
T_{1}\left(N, L_{c}, L_{0}\right)=\frac{L_{c}^{2}}{D_{1}} h\left(N, \frac{L_{0}}{L_{c}}\right)
$$

where

$$
\begin{aligned}
h\left(N, \frac{L_{c}}{L_{0}}\right) \equiv & \sum_{k=1}^{N}\left\{(-1)^{k+1}\left(\begin{array}{l}
N \\
k
\end{array}\right)\right. \\
& \left.\cdot \int_{0}^{+\infty} d t^{\prime}\left[S_{1}\left(t^{\prime} \mid 1, \frac{L_{c}}{L_{0}}\right)\right]^{k}\right\} .
\end{aligned}
$$

When $n$ particles of radius $R$ are within the channel, its length $L_{c}$ reduces to $L_{n}=L_{c}-2(n-1) R$. An effective length $L_{\text {eff }}$ can be estimated through the weighted average

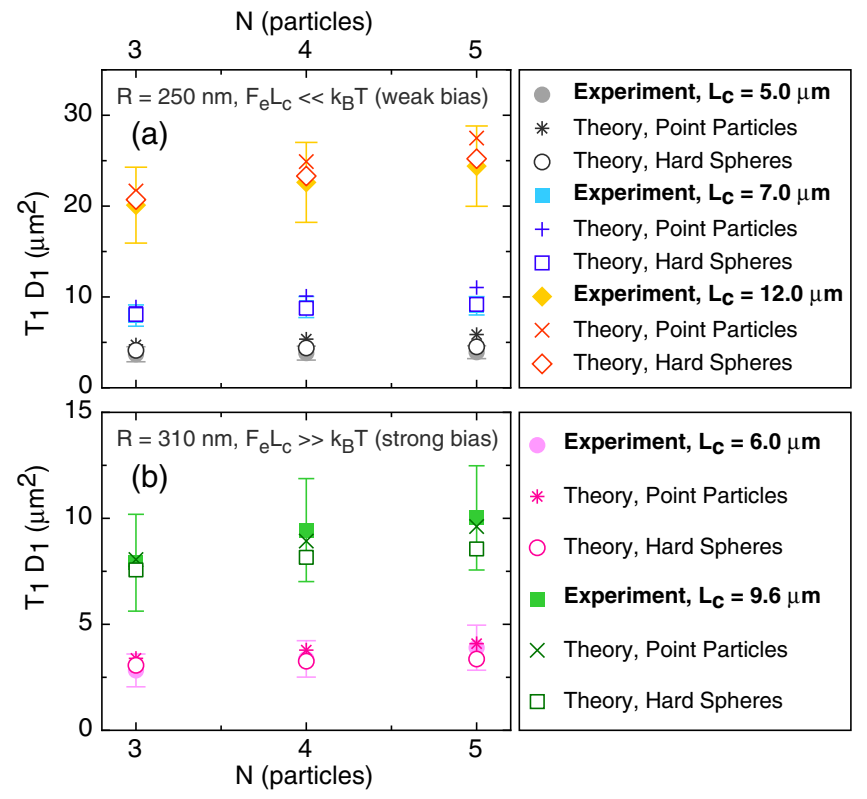

FIG. 3. Dependence of the product of the mean escape time $T_{1}$ and the diffusion coefficient $D_{1}$ on the initial number $N$ of particles in the channel under weak (a) and strong (b) bias conditions. Full symbols are experimental data. Error bars are the propagation of the standard errors for $T_{1}$ and $D_{1}$ recorded in at least 50 experimental independent measurements. Mathematical and open symbols report analytical predictions calculated according to Eqs. (5) and (9), respectively.

$$
\begin{aligned}
L_{\text {eff }}\left(N, L_{c}, L_{0}, \Gamma, R\right) \\
=\frac{\sum_{n=1}^{N}\left[T_{n}-T_{n+1}\right]\left[L_{c}-2(n-1) R\right]}{T_{1}},
\end{aligned}
$$

where

$$
T_{n}\left(N, L_{c}, L_{0}, \Gamma\right)=\int_{0}^{+\infty} d t S_{n}\left(t \mid N, L_{c}, L_{0}, \Gamma\right)
$$

is the mean first passage time of the first $n \leq N$ particles exiting the channel and $\left[T_{n}-T_{n+1}\right]$ is the average time span in which $n$ particles are found in the channel. Excluded-volume corrections are thus effectively taken into account by substituting $L_{c}$ with $L_{\text {eff }}$ in Eq. (5). For $F_{e} L_{c} \ll k_{B} T$ we thus obtain the following expression:

$$
T_{1}\left(N, L_{c}, L_{0}, R\right)=\frac{L_{\text {eff }}\left(N, L_{c}, L_{0}, R\right)^{2}}{D_{1}(R, \Phi)} h\left(N, \frac{L_{0}}{L_{c}}\right) .
$$

The values of $T_{1}$ calculated according to Eq. (9) (open symbols in Fig. 3) are smaller than those obtained by using the pointlike particle approximation in Eq. (3) (mathematical symbols). Intuitively, the effective channel length available to each particle decreases when the particle excluded volume is taken into account.

Equation (9) provides a more accurate description [Fig. 3(a), open symbols] of the experimental data (full symbols) in the presence of a weak external bias with respect to the pointlike particle description (mathematical symbols). Consistently with the Debye range, we define collisions as those events for which the distance between the centers of two neighboring particles becomes smaller than $2.1 R$. Remarkably, we show that an external force $F_{e}=10^{-15} \mathrm{~N}$ is sufficient to drastically decrease the experimentally measured number of interparticle collisions (Fig. S3). Indeed, such a force drags all the particles in one direction, reducing the collision probability. For this reason, in the presence of a strong bias, the pointlike particle predictions are closer to the experimental findings than those of the effective theory with excluded-volume effects [Fig. 3(b)]. Notably, all of the above comparisons between experimental data and theoretical predictions are carried out without fitting parameters, and are only based on the experimental measurements of $L_{0}$ (Fig. S2), $L_{c}, D_{1}$, and $F_{e}$ (see Table S1).

In summary, we have found that the escape process of $N$ particles in single file can be described in terms of the survival probability of the last particle to leave the channel and that the mean escape time of the process scales inversely with the diffusion coefficient of such a particle. By demonstrating that it is sufficient to investigate the diffusion of a single particle, our findings streamline the design of synthetic arrays of channels and pores for applications such as filtering where multiparticle transport under close confinement is paramount. By proving that the 
simple formula $F_{e} L_{c} \simeq k_{B} T\left(F_{e} \simeq 1 \mathrm{fN}\right.$ in our experiments) can be exploited to quantify the effect of an external force on the escape process, our findings help both to rationalize metabolite and drug diffusion across biological membranes under an external force and optimize device geometry in applications involving particle transport in a force gradient. In this respect our theoretical model offers a novel framework for (i) investigating the escape process from widespread biological and synthetic constrictions, and (ii) providing guidelines for the design of microand nanodevices for particle and molecule transport applications.

The authors are indebted to Dr. U. F. Keyser for helpful discussions. S.P. acknowledges the support from the Leverhulme Trust through an Early Career Fellowship (Grant No. ECF-2013-444), the Wellcome Trust through an Institutional Strategic Support Fund (Grant No. SW05377), and the Royal Society through a research grant (Grant No. RG140203). M. P. and E. L. kindly acknowledge funding from the European Research Council under the European Community's Seventh Framework Program (FP7/2007-2013/ERC) Grant Agreement No. 279004.

*Present address: Faculty of Physics, University of Vienna, Boltzmanngasse 5, A-1090 Vienna, Austria.

emanuele.locatelli@univie.ac.at

†matteo.pierno@unipd.it

tbaldovin@pd.infn.it

§orlandini@pd.infn.it

Present address: Department of Biosciences, College of Life and Environmental Sciences, University of Exeter, EX44QD Exeter, United Kingdom. s.pagliara@exeter.ac.uk

[1] P. C. Bressloff and J. M. Newby, Rev. Mod. Phys. 85, 135 (2013).

[2] C. Dekker, Nat. Nanotechnol. 2, 209 (2007).

[3] A. L. Hodgkin and R. D. Keynes, J. Physiol. (London) 128, 61 (1955).

[4] R. Benz, A. Schmid, and G. H. Vos-Scherperkeuter, J. Membr. Biol. 100, 21 (1987).

[5] M. O. Jensen, S. Park, E. Tajkhorshid, and K. Schulten, Proc. Natl. Acad. Sci. U.S.A. 99, 6731 (2002).

[6] J. C. Rasaiah, S. Garde, and G. Hummer, Annu. Rev. Phys. Chem. 59, 713 (2008).

[7] S. Gravelle, L. Joly, F. Detcheverry, C. Ybert, C. CottinBizonne, and L. Bocquet, Proc. Natl. Acad. Sci. U.S.A. 110, 16367 (2013).

[8] G. Portella, T. Polupanow, F. Zocher, D. A. Boytsov, P. Pohl, U. Diederichsen, and B. L. de Groot, Biophys. J. 103, 1698 (2012).

[9] V. Kukla, J. Kornatowski, D. Demuth, I. Gimus, H. Pfeifer, L. V. C. Rees, S. Schunk, K. K. Unger, and J. Kärger, Science 272, 702 (1996).

[10] K. Hahn, J. Kärger, and V. Kukla, Phys. Rev. Lett. 76, 2762 (1996).
[11] J. Kärger, T. Binder, C. Chmelik, F. Hibbe, H. Krautscheid, R. Krishna, and J. Weitkamp, Nat. Mater. 13, 333 (2014).

[12] A. Das, S. Jayanthi, H. S. M. V. Deepak, K. V. Ramanathan, A. Kumar, C. Dasgupta, and A. K. Sood, ACS Nano 4, 1687 (2010).

[13] C. Lutz, M. Kollmann, and C. Bechinger, Phys. Rev. Lett. 93, 026001 (2004).

[14] Q. Wei, C. Bechinger, and P. Leiderer, Science 287, 625 (2000).

[15] B. Lin, M. Meron, B. Cui, S. Rice, and H. Diamant, Phys. Rev. Lett. 94, 216001 (2005).

[16] S. Y. Yang, J.-A. Yang, E.-S. Kim, G. Jeon, E. J. Oh, K. Y. Choi, S. K. Hahn, and J. K. Kim, ACS Nano 4, 3817 (2010).

[17] G.-W. Li, O. G. Berg, and J. Elf, Nat. Phys. 5, 294 (2009).

[18] V. N. Kharkyanen, S. O. Yesylevskyy, and N. M. Berezetskaya, Phys. Rev. E 82, 051103 (2010).

[19] P. M. Richards, Phys. Rev. B 16, 1393 (1977).

[20] T. E. Harris, J. Appl. Probab. 2, 323 (1965).

[21] C. Rödenbeck, J. Kärger, and K. Hahn, Phys. Rev. E 57, 4382 (1998).

[22] T. Chou and D. Lohse, Phys. Rev. Lett. 82, 3552 (1999).

[23] S. Vasenkov and J. Kärger, Phys. Rev. E 66, 052601 (2002).

[24] M. Kollmann, Phys. Rev. Lett. 90, 180602 (2003).

[25] A. M. Berezhkovskii and S. M. Bezrukov, Chem. Phys. 319, 342 (2005).

[26] F. Marchesoni and A. Taloni, Phys. Rev. Lett. 97, 106101 (2006).

[27] L. Lizana and T. Ambjörnsson, Phys. Rev. Lett. 100, 200601 (2008).

[28] A. Taloni and M. A. Lomholt, Phys. Rev. E 78, 051116 (2008).

[29] E. Barkai and R. Silbey, Phys. Rev. Lett. 102, 050602 (2009).

[30] A. Zilman, Biophys. J. 96, 1235 (2009).

[31] P. M. Centres and S. Bustingorry, Phys. Rev. E 81, 061101 (2010).

[32] O. Flomenbom, Phys. Rev. E 82, 031126 (2010).

[33] A. Ryabov and P. Chvosta, Phys. Rev. E 83, 020106 (2011).

[34] G. Suárez, M. Hoyuelos, and H. O. Mártin, Phys. Rev. E 88, 022131 (2013).

[35] E. Locatelli, F. Baldovin, E. Orlandini, and M. Pierno, Phys. Rev. E 91, 022109 (2015).

[36] B. H. Lin, B. X. Cui, J. H. Lee, and J. Yu, Europhys. Lett. 57, 724 (2002).

[37] U. Siems, C. Kreuter, A. Erbe, N. Schwierz, S. Sengupta, P. Leiderer, and P. Nielaba, Sci. Rep. 2, 1015 (2012).

[38] G. Coupier, M. Saint Jean, and C. Guthmann, Phys. Rev. E 73, 031112 (2006).

[39] L. Dagdug, A. M. Berezhkovskii, and S. M. Bezrukov, J. Chem. Phys. 137, 234108 (2012).

[40] X. Xu, B. Lin, B. Cui, A. R. Dinner, and S. A. Rice, J. Chem. Phys. 132, 084902 (2010).

[41] E. Wonder, B. Lin, and S. A. Rice, Phys. Rev. E 84, 041403 (2011).

[42] B. Lin, B. Cui, X. Xu, R. Zangi, H. Diamant, and S. A. Rice, Phys. Rev. E 89, 022303 (2014).

[43] S. Bleil, P. Reimann, and C. Bechinger, Phys. Rev. E 75, 031117 (2007). 
[44] J. E. Curtis, B. A. Koss, and D. G. Grier, Opt. Commun. 207, 169 (2002).

[45] D. Grier, Nature (London) 424, 810 (2003).

[46] M. Padgett and R. Bowman, Nat. Photonics 5, 343 (2011).

[47] M. Padgett and R. Di Leonardo, Lab Chip 11, 1196 (2011).

[48] J. C. Crocker and D. G. Grier, J. Colloid Interface Sci. 179, 298 (1996).

[49] S. Pagliara, C. Schwall, and U. F. Keyser, Adv. Mater. 25, 844 (2013).

[50] S. L. Dettmer, U. F. Keyser, and S. Pagliara, Rev. Sci. Instrum. 85, 023708 (2014).

[51] S. L. Dettmer, S. Pagliara, K. Misiunas, and U. F. Keyser, Phys. Rev. E 89, 062305 (2014).

[52] S. Pagliara, S. L. Dettmer, and U. F. Keyser, Phys. Rev. Lett. 113, 048102 (2014).
[53] S. Pagliara, C. Chimerel, R. Langford, D. G. A. L. Aarts, and U. F. Keyser, Lab Chip 11, 3365 (2011).

[54] S. Pagliara, S. L. Dettmer, K. Misiunas, L. Lea, Y. Tan, and U. F. Keyser, Eur. Phys. J. Spec. Top. 223, 3145 (2014).

[55] See Supplemental Material http://link.aps.org/supplemental/ 10.1103/PhysRevLett.117.038001 for a sample video of our experiments, details about the experiments, and theoretical models, including Refs. [56-58].

[56] P. M. Bungay and H. Brenner, Int. J. Multiphase Flow 1, 25 (1973).

[57] E. Barkai and R. Silbey, Phys. Rev. E 81, 041129 (2010).

[58] N. Leibovich and E. Barkai, Phys. Rev. E 88, 032107 (2013).

[59] J. D. Chodera and V. S. Pande, Phys. Rev. Lett. 107, 098102 (2011). 